\title{
Instrumentos de gestión de áreas naturales protegidas y participación comunitaria
}

\section{Managment tools of natural protected areas and community participation}

\author{
Julisa Guadalupe Cabrera-Borraz ${ }^{1}$ \\ Lucinda Arroyo-Arcos ${ }^{2}$ \\ Romano Gino Segrado-Pavón ${ }^{3}$ \\ Orlando Colín-Olivares ${ }^{4}$ \\ Universidad de Quintana Roo, México
}

\begin{abstract}
Resumen
El objetivo del estudio fue analizar la gestión del territorio a partir de los instrumentos de gestión y la participación comunitaria en la Reserva de la Biosfera de Sian Ka'an, Quintana Roo, México. El método para la obtención de información consistió en la revisión documental referente a los instrumentos dictados por la legislación ambiental mexicana para la gestión de Áreas Naturales Protegidas, y la aplicación de 30 guías de entrevista semi-estructuradas con base a la teoría de la acción colectiva, a actores clave de la comunidad Punta Allen, ubicada dentro de la RBSK. Como principales hallazgos se identificaron dos actividades económicas directamente vinculadas a los instrumentos de gestión, que determinan la participación comunitaria y el aprovechamiento de los recursos naturales.
\end{abstract}

Palabras clave: Instrumentos de gestión, Áreas Naturales Protegidas, participación comunitaria

1 Maestrante en Gestión Sustentable del Turismo, Universidad de Quintana Roo, Av. Andrés Quintana Roo s/n, con 110 sur, Cozumel Quintana Roo, México. Correo electrónico: julicabo_18@hotmail.com

2 Profesora-investigadora de la Universidad de Quintana Roo, México. Correo electrónico: lucindaarroyo@, hotmail.com

3 Profesor-investigador de la Universidad de Quintana Roo, México. Correo electrónico: romanogino@hotmail.com

4 Maestrante en Gestión Sustentable del Turismo, Universidad de Quintana Roo, México. Correo electrónico: colinolivareso@gmail.com

Este artículo corresponde a la ponencia presentada en el 35th Conference of Latin American Geographers realizada en San José, Costa Rica del 20 al 22 de mayo del 2018. 


\begin{abstract}
The aim of this study was to analyze the management of the territory by the management tools and the community participation at the Sian Ka'an Biosphere in Quintana Roo, Mexico. The method used to obtain the data, was a literary review of tools given by the Mexican environmental legislation for the management of natural protected areas, also 30 semi-structured interviews were carried out with key actors based on the collective action theory in Punta Allen, located into the Sian Ka' an Biosphere. The main findings include the detection of two economic activities related with the management tools, and the identified activitities determine the community participation and the use of natural resources.
\end{abstract}

Keywords: Tools management, natural protected areas, community participation.

\title{
Introducción
}

La política ambiental en México propone como principal directriz la necesidad de gestión de los recursos naturales y el desarrollo sustentable (Pérez, 2010), para lo cual se plantea una serie de instrumentos legales de tipo ecológico, social, y económico, que conducen a la gestión sustentable de los sistemas ambientales. Entre ellos, las Áreas Naturales Protegidas (ANP), que se han convertido en el instrumento cardinal de política ambiental para la conservación de los recursos naturales y de la biodiversidad, con ellas se ha logrado la conservación de ambientes naturales.

Sin embargo, el decreto de éstas, además de generar la delimitación y protección del espacio, también genera transformaciones en la configuración social.

Otros aspectos criticados en la instauración de ANP, son la falta de participación social y la carencia de articulación entre las comunidades locales e instituciones rectoras encargadas de la administración (López, Bello, Estrada, Brunel \& Ramírez, 2012). Cuando esta situación es disfuncional provoca que los instrumentos de apoyo de gestión de ANP no sean adoptados o respetados por las comunidades enclavadas, provocando el colapso de estos sistemas y por ende, que no se cumplan con los objetivos estipulados en el decreto. De acuerdo a Ostrom (2000), las estrategias implementadas en espacios de propiedad común, en este caso los recursos naturales de las ANP, tenderán a tener mayor adopción cuando son diseñadas por los propios usuarios a diferencia de reglas impuestas por agentes externos.

Según Mirabal \& Flores (2016), las comunidades locales son actores primordiales en la gestión de las ANP, ya que como principales usuarios fortalecen la conciencia y el empoderamiento del espacio, promoviendo 
Julisa Guadalupe Cabrera-Borraz, Lucinda Arroyo-Arcos, Romano Gino Segrado-Pavón, Orlando Colín-Olivares. Instrumentos de gestión de áreas naturales protegidas y participación comunitaria

la creación de estrategias para el aprovechamiento y conservación de los recursos naturales.

El grado de participación e involucramiento de la comunidad se delimita según la categoría del ANP; en México existen 182 ANP (CONANP 2017), de las cuales 45 son Reservas de la Biosfera, 40 Áreas de Protección de Flora y Fauna, 18 Santuarios, 8 Áreas de Protección de Recursos Naturales, 5 Monumentos Naturales y 66 Parques Nacionales. El estado de Quintana Roo, posee 17 de éstas, y una de las más importantes es la Reserva de la Biosfera de Sian Ka'an (RBSK), por su alcance territorial.

Una de las características más importantes de la RBSK, es que forma parte de la Red Internacional del Hombre y la Biosfera (MaB por sus siglas en inglés), este programa promueve el binomio "conservación y participación" de manera armoniosa, con la finalidad de fomentar el aprovechamiento y el manejo sustentable de los recursos naturales. Sin embargo, según Brenner (2010), la RBSK experimenta una serie de problemas que obstaculizan la gestión que van desde la amenaza del equilibrio ecológico, así como la falta de integración social entre pobladores, la carencia de recursos humanos y financieros que no permiten el manejo adecuado, y el uso de recursos naturales de manera inapropiada por parte de los ejidatarios.

Considerando el contexto planteado, la investigación surgió con el objetivo de analizar la gestión del territorio a partir de los instrumentos de gestión y la participación comunitaria en la Reserva de la Biosfera de Sian Ka'an, Quintana Roo, México.

\section{Marco teórico-conceptual}

La participación alude a la intervención de los individuos y grupos sociales en consultas, discusiones, propuestas y todo tipo de actividades interrelacionadas para el progreso de una comunidad (Guillen, Sáenz, Badii, \& Castillo, 2009). Según Ziccardi (1998) existen diferentes formas de participación como: la política, social, ciudadana, y comunitaria.

La participación comunitaria se considera fundamental para garantizar la sustentabilidad, se basa en involucrar a la población en la toma de decisiones, a partir del reconocimiento de problemas en conjunto y el planteamiento de soluciones (Artigas, Ramos, \& Vargas, 2014). Para que este proceso se lleve a cabo es necesario que la población se sienta parte del territorio, en otras palabras que exista el sentimiento de pertenencia. 
Además de la participación de la comunidad en la gestión del territorio como bien comunal, es importante la participación de distintos actores y la consideración de planificaciones previas para contribuir en mejora de la calidad de vida de los habitantes (Morúa, 2010).

Desde la perspectiva de Ostrom (2011) en la gestión de bienes comunales, es necesario crear un sistema colectivo de gobernanza que incorpore los distintos intereses de forma constructiva, las necesidades y las potencialidades para alcanzar los objetivos y beneficios en común (Santana, Filho \& Rocha, 2015), lo que implica la implementación niveles de uso del espacio, la distribución equitativa de beneficios y monitoreo a fin de reducir los efectos negativos que pudieran surgir (Segrado, Serrano, Cruz \& Juan, 2013; Ostrom, 2011).

Desde el marco jurídico ambiental, la federación promueve la participación de la sociedad en la administración y el manejo de las ANP (LGEEPA, 2012). En ocasiones, resulta complicado cumplir con lo estipulado en la legislación, e incluso hay casos donde las áreas protegidas no son aceptadas por la población local (Schmidt, 2010; Villalobos, 2000) porque ya existían asentamientos previos al decreto y se requiere de todo un proceso de concientización y aplicación de regulaciones territoriales y administrativas para que sean aceptadas. Por eso es importante de delimitar espacialmente las zonas de uso y no uso (Paz, 2008).

En el artículo 47 BIS de la LGEEPA estas zonas se establecen según la vocación y características del espacio, el área se divide en zonas núcleo y zonas de amortiguamiento. Este proceso de zonificación se considera un instrumento técnico de planeación, y regularmente se realiza después de un estudio de ordenamiento territorial, ya que en esencia pretende compatibilizar el aspecto social y el aprovechamiento de los recursos naturales presentes (Segrado, Arroyo, \& Amador, 2010).

\section{Área de estudio}

La Reserva de la Biosfera de Sian Ka'an, puerta del cielo o donde nace el cielo según la interpretación de la lengua maya (McInnes, Kenza Ali, \& Pritchard, 2017) se localiza en el litoral central del estado de Quintana Roo, México (ver figura 1). Tiene una superficie de 528,147 hectáreas entre los municipios de Tulum y Felipe Carrillo Puerto (CONANP, 2014). Fue establecida como Área Natural Protegida oficialmente el 20 de enero 
Julisa Guadalupe Cabrera-Borraz, Lucinda Arroyo-Arcos, Romano Gino Segrado-Pavón, Orlando Colín-Olivares. Instrumentos de gestión de áreas naturales protegidas y participación comunitaria

de 1986 mediante un decreto presidencial publicado en el Diario Oficial de la Federación (DOF), el mismo año se incorporó a la lista de las Reservas de la Biosfera del Programa el Hombre y la Biosfera (MaB, por sus siglas en inglés) de la UNESCO, en 1987 fue declarada como Sitio del Patrimonio Mundial como bien natural y en el año 2003 fue inscrita en la lista de Humedales de Importancia Internacional por la Convención Ramsar.

Figura 1. Área de estudio

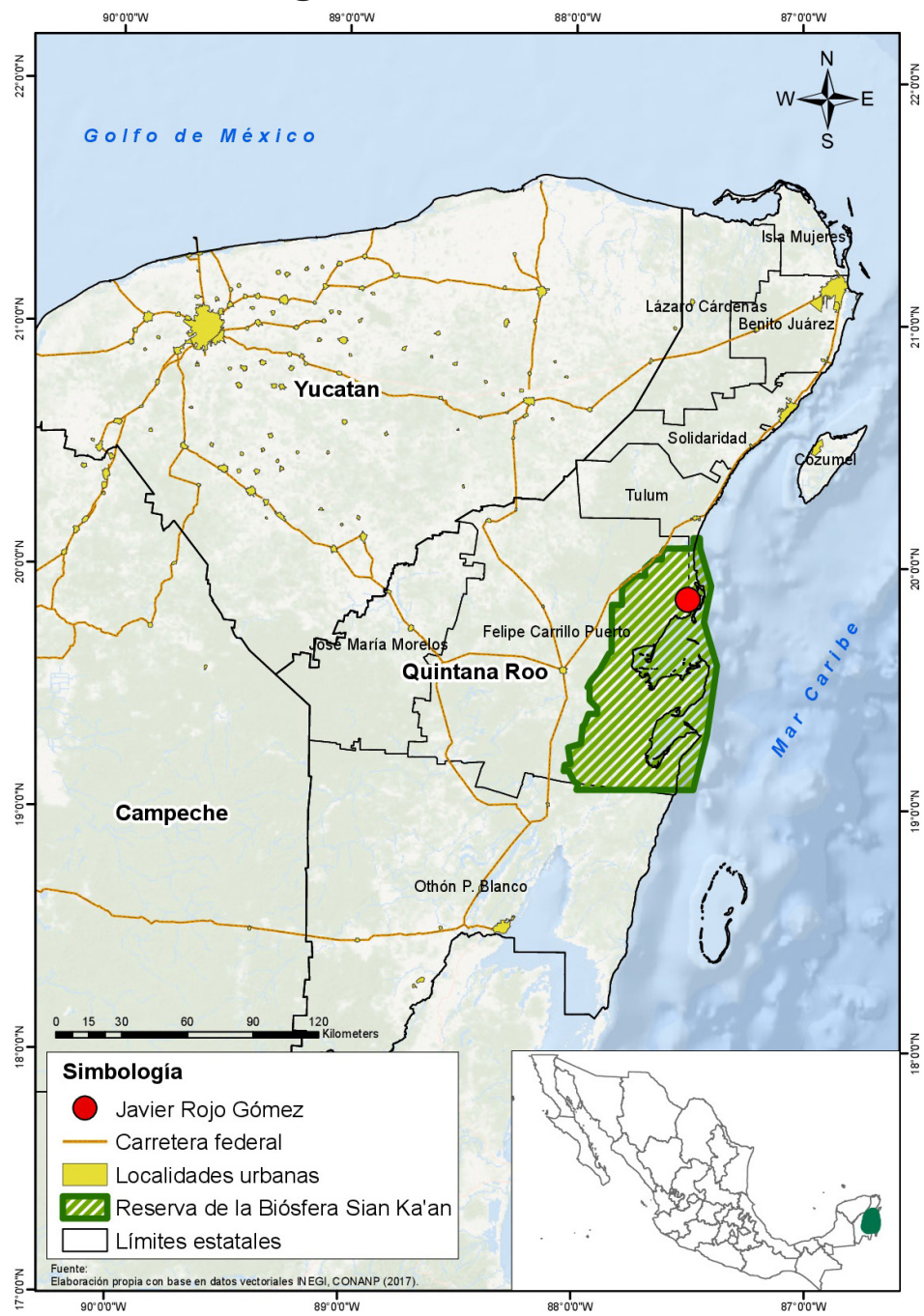

Fuente: elaboración propia, datos vectoriales obtenidos de INEGI, y CONANP, 2017. 
El área se ubica en una planicie kárstica parcialmente emergida que desciende gradualmente hacia el mar (DOF, 2015), este gradiente es el que permite la formación de ecosistemas como selvas medianas, selvas bajas, marismas, manglares, lagunas y arrecifes por mencionar algunos, además albergan especies de flora y fauna endémicas de la región, amenazadas, sujetas a protección especial o en su caso en peligro de extinción según la Norma Oficial Mexicana NOM-059-SEMARNAT-2010.

La RBSK cuenta con un Programa de Manejo (CONANP, 2014) como instrumento rector de planeación y regulación, en el cual se establece la zonificación de la RBSK, que consta de tres subzonas núcleo en las cuales se restringe la realización de actividades humanas, la zona de amortiguamiento se subzonifica en múltiples subzonas de preservación, de aprovechamiento sustentable de recursos pesqueros, de uso público, de asentamientos humanos, de recuperación y de aprovechamiento especial.

Referente a la zona de asentamientos humanos, la RBSK está habitada por aproximadamente 1000 personas, el 46.9\% de esta población se encuentra asentada en Punta Allen. Esta comunidad se sitúa geográficamente en $19^{\circ} 47^{\prime} 59^{\prime \prime} \mathrm{N} 87^{\circ} 28^{\prime} 35^{\prime \prime} \mathrm{O}$, se ubica dentro del polígono de la RBSK, en la zona de amortiguamiento (subzona de asentamientos humano), abarca una superficie de 28.9286 hectáreas, habitan 469 personas y 128 viviendas particulares (Instituto Nacional de Estadística y Geografía, 2010). Debido a su ubicación en el litoral, la comunidad tiene la ventaja en el aprovechamiento de los recursos naturales y paisajísticos, sus principales actividades económicas son la pesca y el turismo.

\section{Marco metodológico}

Para analizar la gestión del territorio a partir de los instrumentos de gestión y la participación comunitaria en la Reserva de la Biosfera de Sian Ka'an, Quintana Roo, México se optó por realizar una investigación con enfoque cualitativo, de corte etnográfico y carácter descriptivo. Las investigaciones cualitativas se caracterizan por utilizar el método inductivo, su principal objeto es explorar, además de proporcionar profundidad y riqueza a los datos (Cortés, 2012). Los estudios etnográficos de corte transversal, abordan el objeto de estudio (comunidades) con miras de comprender su interacción con un amplio contexto, en un tiempo determinado (Murillo y Martínez, 2010). En cuanto a los estudios descriptivos, se caracterizan 
Julisa Guadalupe Cabrera-Borraz, Lucinda Arroyo-Arcos, Romano Gino Segrado-Pavón, Orlando Colín-Olivares. Instrumentos de gestión de áreas naturales protegidas y participación comunitaria

por describir diversos aspectos del fenómeno o comunidad a estudiar (Hernández, Fernández y Baptista, 2010).

La investigación se desarrolló en las siguientes etapas:

1. Revisión bibliográfica y diseño metodológico

2. Recopilación de datos cartográficos y aplicación de instrumentos de recolección de datos

3. Codificación y procesamiento de información en software Atlas. Ti versión 8 , y análisis de información recolectada

En la primera etapa se llevó a cabo la revisión y compilación de documentos oficiales que involucran los documentos rectores de la gestión de la RBSK, como el decreto de creación del área protegida, el programa de manejo, publicaciones en el Diario Oficial de la Federación referentes a las concesiones otorgadas para el aprovechamiento de la RBSK, y las actas constitutivas de las sociedades cooperativas de servicios turísticos y la sociedad cooperativa de servicios pesqueros como principales actores de aprovechamiento del área.

Se diseñó el instrumento de recolección de datos, el cual consistió en una guía de entrevista estructurada en cinco secciones, la primera corresponde a aspectos generales del entrevistado y las cuatro restantes a las categorías de la teoría de acción colectiva de Ostrom (sistema de recursos, sistemas de gobernanza, unidades de recursos, usuarios).

La segunda etapa se realizó en verano de 2017, se aplicaron 30 entrevistas por la técnica bola de nieve a personas residentes de la comunidad. La muestra no es representativa y es correspondiente a la cantidad sugerida por Hernández, Fernández, \& Baptista (2010) para estudios de corte etnográfico. Asimismo e reunieron datos cartográficos para la elaboración de mapas.

En la tercera etapa se transcribieron los audios de entrevistas en verbatim, y se cargaron el software Atlas. Ti versión 8 , se codificaron de acuerdo a las categorías de las secciones de la guía anteriormente mencionadas, y posteriormente se crearon informes para analizar la información. 


\section{Resultados}

Los instrumentos de gestión y la participación comunitaria se explican a través de las categorías analizadas de la teoría de la acción colectiva.

En los sistemas de recursos naturales, se identificaron tres actividades económicas, la pesca de langosta y el turismo de naturaleza son las principales, y en menor escala la pesca deportiva. En la pesca de langosta participan los fundadores de la Sociedad Cooperativa de Producción Pesquera "Pescadores de Vigía Chico", S. C.L desde 1968, y nadie más tiene acceso a menos que sea primogénito de un socio directamente, en cuanto al turismo de naturaleza, existen seis cooperativas de servicios turísticos y cualquier persona residente de la comunidad que cumpla con los reglamentos dictados en las actas constitutivas puede participar en la actividad. Referente a la pesca deportiva esta, no es directamente encabezada por la comunidad.

Estas actividades están delimitadas según la zonificación establecida en el Programa de Manejo de la RBSK, siendo éste el principal instrumento que regula las directrices de administración y manejo del área protegida. En él se establecen las actividades y acciones permitidas en el área, es decir, que sean compatibles con la vocación de conservación. En la zonificación (ver figura 2) se dispusieron de tres subzonas núcleo en las cuales se restringe la realización de actividades humanas, y una zona de amortiguamiento subzonifica en múltiples subzonas de preservación, de aprovechamiento sustentable de recursos pesqueros, de uso público, de asentamientos humanos, de recuperación, y de aprovechamiento especial. 
Julisa Guadalupe Cabrera-Borraz, Lucinda Arroyo-Arcos, Romano Gino Segrado-Pavón, Orlando Colín-Olivares. Instrumentos de gestión de áreas naturales protegidas y participación comunitaria

Figura 2. Zonificación de la Reserva de la Biosfera de Sian Ka'an

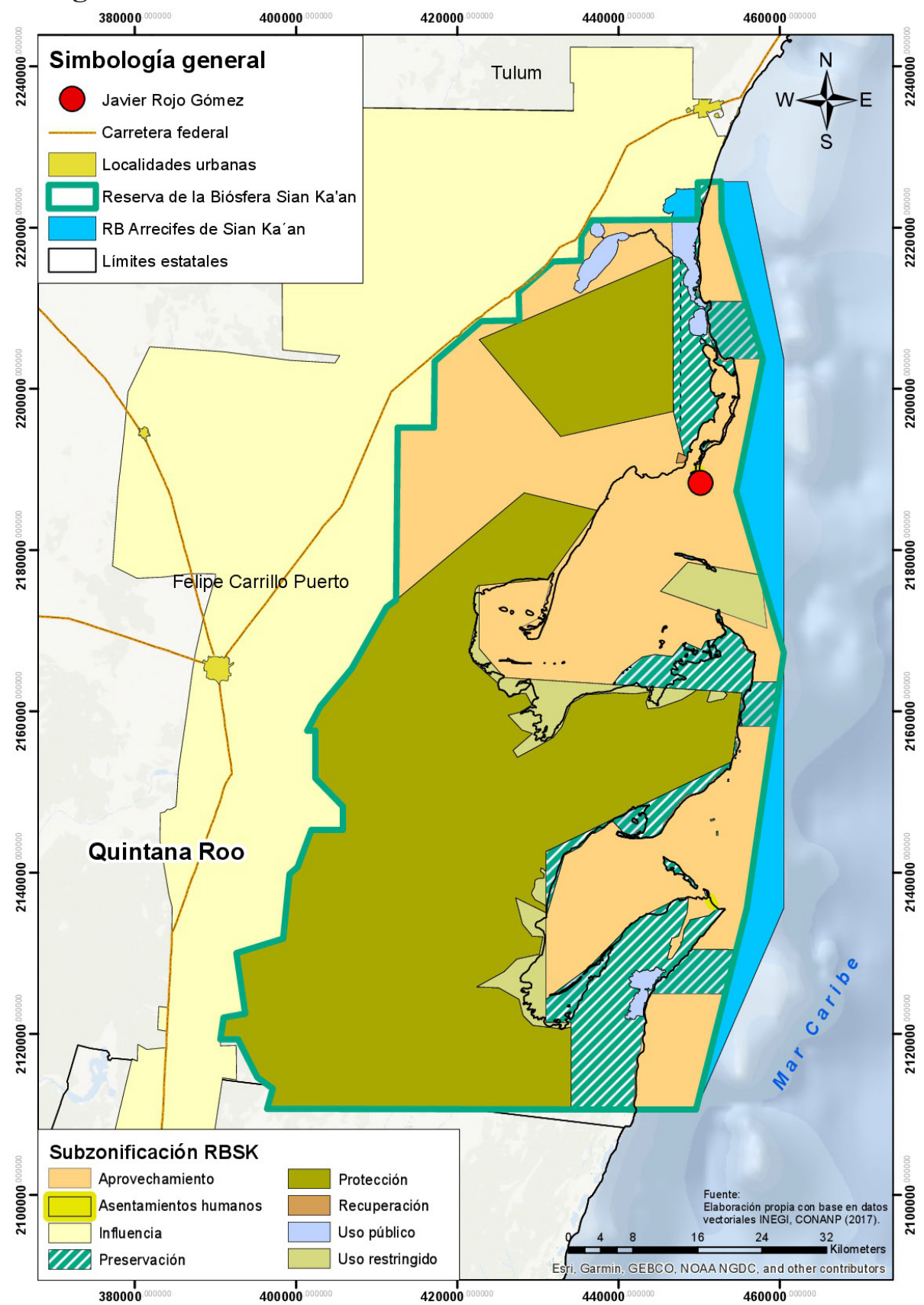

Fuente: elaboración propia, datos vectoriales obtenidos de INEGI, y CONANP, 2017. 
En lo que se refiere a la subzona de asentamientos humanos, la RBSK está habitada por aproximadamente 1000 personas, la comunidad más representativa es Punta Allen, en ella habita el $46 \%$ de la población total, además capta aproximadamente el $90 \%$ de turistas que arriba a la RBSK.

En cuanto a las unidades de recursos, pueden dividirse en dos sectores, el de pesca de langosta y el de turismo de naturaleza. La pesca de langosta tiene una concesión para todos los socios, en la zona ubicada frente a Punta Xamach hasta Punta Pájaros, incluyendo la Bahía de Ascensión (ver figura 3), esta concesión es subdividida por georreferenciación en parcelas marinas y a cada socio de la cooperativa le corresponde determinada fracción, este modelo de parcelación inició en 1978 y actualmente es un modelo reconocido de pesca sustentable. 
Julisa Guadalupe Cabrera-Borraz, Lucinda Arroyo-Arcos, Romano Gino Segrado-Pavón, Orlando Colín-Olivares. Instrumentos de gestión de áreas naturales protegidas y participación comunitaria

Figura 3. Parcelación marina para el aprovechamiento del recurso pesquero

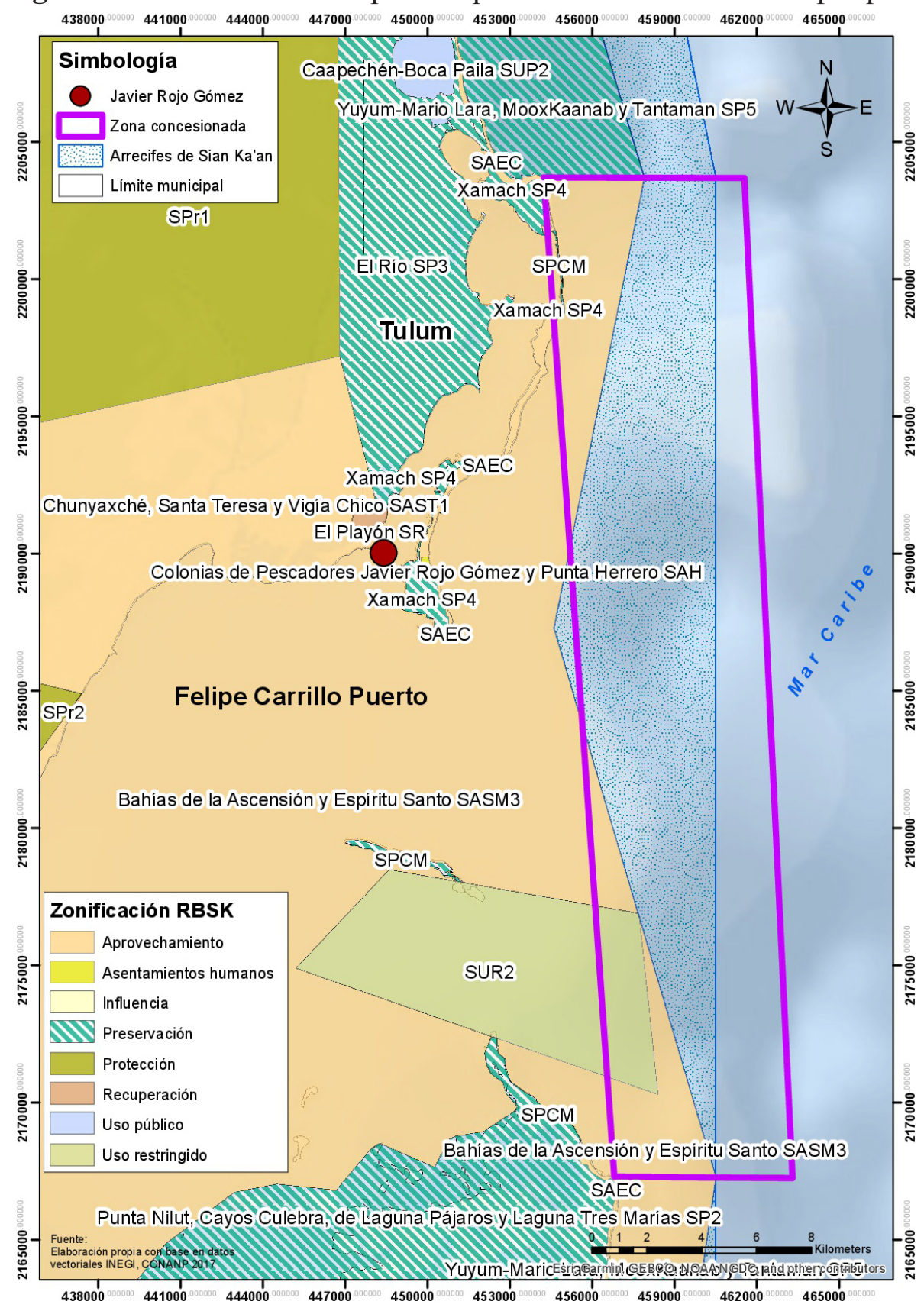

Fuente: elaboración propia, datos vectoriales obtenidos de INEGI, y CONANP, 2017. 
En la concesión publicada en el Diario Oficial de la Federación (1994), se prohíben actividades como la alteración de zonas de desove, introducción de especies de flora y fauna sin autorización previa, el uso de explosivos que dañen a las especies marinas y redes de arrastre.

Además del acompañamiento de las medidas de regulación que implican la adopción de instrumentos legales federales como el Programa de Manejo de la Reserva, Normas Mexicanas (NOM-006-PESC-1993) para regular las artes de pesca y medidas de la langosta apta para comercializar, así como también los periodos de pesca y veda de la langosta, y también se acatan estrictamente al reglamento interno de la cooperativa que en caso de infringir, el socio es acreedor a una sanción monetaria, expulsión temporal e incluso expulsión definitiva de la cooperativa o de la comunidad, según sea la gravedad de la falta (J. Pereira, comunicación personal, 28 de junio, 2017).

En cuanto al turismo, las sociedades cooperativas tienen concesiones otorgadas de acuerdo a los usos permitidos en las subzonas de la zona de amortiguamiento. Principalmente se hace uso de los arrecifes de coral, para actividades como avistamiento de fauna marina, esnórquel.

Para poder llevar a cabo esta actividad, se deben seguir algunas medidas, una de ellas es obligatoria y es que nadie puede realizar esnórquel en la barrera de arrecifes de coral, sin el acompañamiento de un guía en una embarcación con un grupo que no exceda los seis visitantes, al llevar a cabo la actividad se debe mantener una distancia aproximada dos metros de los corales, usar chalecos salvavidas para evitar la manipulación y daño de corales, por mencionar algunos puntos establecidos por la legislación ambiental y el reglamento interno de las sociedades cooperativas turísticas.

En cuanto a los sistemas de gobernanza, las bases de la organización de la comunidad son las sociedades cooperativas, están juegan un papel importante en la comunidad. A continuación se presentan los datos generales de la sociedades cooperativas de la comunidad (ver tabla 1). 
Julisa Guadalupe Cabrera-Borraz, Lucinda Arroyo-Arcos, Romano Gino Segrado-Pavón, Orlando Colín-Olivares. Instrumentos de gestión de áreas naturales protegidas y participación comunitaria

Tabla 1. Sociedades cooperativas de servicios pesqueros y turísticos en Punta Allen

\begin{tabular}{|c|c|c|}
\hline Año de creación & Nombre & Tipo de actividades \\
\hline 1986 & $\begin{array}{l}\text { Soc. Coop. de Servicios Pesqueros Vigía } \\
\text { Chico }\end{array}$ & Producción de langosta \\
\hline 1994 & $\begin{array}{l}\text { Soc. Coop. de Servicios Turísticos } \\
\text { "Punta Alem", S.C.L }\end{array}$ & \multirow{5}{*}{$\begin{array}{l}\text { Esnórquel, observación } \\
\text { de fauna marina (delfines } \\
\text { y tortugas), observación } \\
\text { de aves en Cayos. }\end{array}$} \\
\hline 1997 & $\begin{array}{l}\text { Soc. Coop. de Servicios Turísticos } \\
\text { Vigía Grande S.C.L de Responsabilidad } \\
\text { Limitada }\end{array}$ & \\
\hline 1998 & $\begin{array}{l}\text { Soc. Coop. de Servicios Turísticos Los } \\
\text { Gaytanes }\end{array}$ & \\
\hline 1999 & $\begin{array}{l}\text { Soc. Coop. de Servicios Turísticos Las } \\
\text { Boyas }\end{array}$ & \\
\hline 2009 & $\begin{array}{l}\text { Soc. Coop. de Servicios Turísticos } \\
\text { Nativos de la Bahía de R.L de C.V }\end{array}$ & \\
\hline 2014 & $\begin{array}{l}\text { Soc. Coop. de Responsabilidad Limitada } \\
\text { de Capital Variable "Orquídeas de Sian } \\
\text { Ka'an" de R.L de C.V }\end{array}$ & $\begin{array}{l}\text { Senderismo, ciclismo, } \\
\text { kayakismo, observación } \\
\text { de aves. }\end{array}$ \\
\hline
\end{tabular}

Fuente: elaboración propia con base a las actas constitutivas de las sociedades cooperativas.

Durante el desarrollo de estas cooperativas se manifestaron problemas sociales dentro de la comunidad, provocados principalmente por la competencia entre cooperativas, debido a esa problemática aproximadamente en 2003 se acordó crear una "alianza de cooperativas" (B. Caamal, comunicación personal, 03 de julio, 2017) integrada por presidentes de cuatro cooperativas turísticas (Vigía Grande, Gaytanes, Punta Alem, y las Boyas), algunas de las funciones que realizan es estandarizar las actividades, los precios de tours y alimentos, y cuestiones de toma de decisiones sobre la infraestructura vial, medio ambiente, limpieza de playas, colocación de boyas, elaboración o modificación del reglamento interno, visitas a la presidencia municipal o a la dirección de la CONANP, según sea el caso. En sí esta alianza ha permitido mantener el orden al estandarizar el servicio y ha permitido trabajar en equipo por el bien común de cada integrante de las cooperativas, así como de la población local.

A partir de las sociedades cooperativas, se regula de manera interna el aprovechamiento de los recursos naturales, mediante las buenas prácticas ambientales y reglamentos administrativos internos. De acuerdo a las 
entrevistas el aprovechamiento de los recursos a permitido la revalorización de la reserva y es así como por iniciativa propia se han instaurado medidas de regulación.

"nosotros hemos conservado todo esto, nos hemos autocriticado por decirlo así y autogobernado disciplinadamente" (C. Chog, comunicación personal, 10 de julio, 2017).

Cuando alguien infringe en el reglamento interno, son sancionados, por ejemplo la primera es una amonestación verbal, la segunda una sanción económica que consiste en la suspensión del trabajador por una semana sin derecho a salario y tampoco es aceptado en otra cooperativa (J. Pereira, comunicación personal, 28 de junio, 2017). Para el caso de los reglamentos establecidos por la SCPVC se toman medidas extremas como la expulsión definitiva de la sociedad.

En cuanto a los gestores gubernamentales y organizaciones de apoyo, se identificaron a dos principalmente, a la Comisión Nacional de Áreas Naturales Protegidas que es la dependencia creada con el objetivo principal de las ANP, y a la sociedad civil Amigos de Sian Ka'an; aunque cabe mencionar que entre estas, existe confusión por parte de la población y no logran diferenciar en la administración y función de cada una.

Tres de los entrevistados, confunden las administraciones o desconocen de sus funciones, estas lagunas de conocimiento y la relación hacen evidente la falta de correspondencia entre estás y la comunidad, situación que debe ser atendida para mejor atender las necesidades del pueblo, y gestionar adecuadamente la RBSK. El 26\% considera que el trabajo realizado no es suficiente, ya que consideran que debe haber mayor acercamiento y colaboración con la comunidad, para trabajar y tomar decisiones en conjunto.

... les hace falta más presencia, [...] involucrar más a las cooperativas, $[\ldots]$ involucrar a todas a que participen. (T. Mendoza, comunicación personal, 10 de julio, 2017).

En cuanto a la última categoría de análisis, correspondiente a los usuarios, el aprovechamiento de la RBSK data desde principios del siglo XIX (guerra de castas) es decir previo al decreto del ANP. En esas fechas los ingleses y la población local explotaba los recursos a través de la caza de 
Julisa Guadalupe Cabrera-Borraz, Lucinda Arroyo-Arcos, Romano Gino Segrado-Pavón, Orlando Colín-Olivares. Instrumentos de gestión de áreas naturales protegidas y participación comunitaria

manatí, tortuga, y lagarto (D. Isabel, comunicación personal, 29 de junio de 2017). A principios de los años cincuenta el aprovechamiento torno a la extracción de chicle y producción de copra (P. Angélica, comunicación personal, 02 de julio de 2017), en 1960 ambas actividades decayeron. En 1968 surgió la pesca de langosta que hasta la fecha persiste, sin embargo posterior al decreto surgieron medidas de regulación que modificaron las formas de vida de la comunidad y el aprovechamiento de los recursos naturales, sin embargo con el paso del tiempo y concientización se adoptaron las medidas implementadas, y actualmente son los usuarios los pioneros en la regulación y distribución de recursos. Como se mencionó, también realizan turismo de bajo impacto, esta última actividad comenzó en 1994 aproximadamente.

Los ecosistemas de la RBSK han permitido que la población se emplee en diversos medios de vida, esta es una ventaja para la población local y una característica importante de la RBSK. Ya que la población no depende de una sola actividad como fuente de ingreso, es decir que hay alternancia en las actividades, el periodo de veda de langosta consta del 01 de marzo al 30 de junio, y en cuanto al turismo es una actividad que puede realizarse durante todo el año, la última cifra de arribo a la RBSK fue de 160,522 visitantes en el año 2017, que en su mayoría fueron captados por la comunidad, por prestadores de servicios de las sociedades cooperativas.

Según la población local esta alternancia de actividades ha promovido la mejora en su calidad de vida, y es que también es importante destacar que estos beneficios son derivados debido al sistema de cooperativas de la comunidad, que permite permear los ingresos económicos al ser ellos los principales gestores del espacio.

\section{Discusión de resultados}

A pesar de los objetivos de establecimiento de áreas naturales protegidas como herramientas de conservación y promoción de desarrollo sustentable, es difícil cumplir con éstos. Como mencionan Schmidt (2010) y Villalobos (2000) las declaratorias no son aceptadas por la población local por las modificaciones en sus actividades y la instauración de regulaciones dictadas por la legislación ambiental.

En la RBSK esta situación no es ajena, y se experimentó en 1986 con el decreto, puesto que desde 1970 oficialmente ya existía la comunidad asentada dentro del área. Al principio la declaratoria no fue aceptada por 
las regulaciones surgidas respecto al aprovechamiento del recurso pesquero principalmente, sin embargo después de un largo proceso de concientización por parte de la Comisión Nacional de Áreas Naturales Protegidas, y organizaciones no gubernamentales como Amigos de Sian Ka'an A.C, las medidas fueron aceptadas. Coincidiendo con la aseveración de Morúa (2010), quien indica que en la gestión del territorio es importante la participación de la comunidad en colaboración con los actores encargados en diferentes niveles.

La aceptación de políticas de gestión en la comunidad, radica de la conciencia generada a través de los años, es decir al ser usuarios directos y beneficiarse del aprovechamiento de los recursos, ha permitido crear estrategias a nivel comunidad para el uso sustentable de los recursos naturales. Un factor determinante en el éxito en la gestión de la RBSK, ha sido la organización de la comunidad en sociedades cooperativas, de acuerdo con Ostrom (2000) quien menciona que cuando las estrategias son creadas por los propios usuarios son propensas a tener mayor aceptación.

También es importante resaltar que en conjunto, el sistema de parcelas, la organización de la comunidad y la compaginación de instrumentos legales tiene una serie de beneficios biológicos, económicos y sociales para la RBSK (WWF, 16). De acuerdo con Villanueva, y otros (2017) algunos puntos importantes de resaltar en la reserva, es que autoorganización de la comunidad permite regular el acceso a los recursos y se mejora la gestión, así como la zonificación de las parcelas influye en la distribución de beneficios para la población, y la correlación entre federación y el sistema de cooperativa mejora la efectividad del aprovechamiento del espacio.

\section{Referencias}

Artigas Pérez, E., Ramos Rodríguez, A. E., \& Vargas Rodríguez, H. (2014). La participación comunitaria en la conservación del medio ambiente: clave para el desarrollo local sostenible. DELOS Desarrollo Local Sostenible, 1-21.

CONANP. (2014). Programa de Manejo Complejo Sian Ka'an: Reserva de la Biosfera Sian Ka'an, Área de Protección de Flora y Fauna Uaymil y Reserva de la Biosfera Arrecifes de Sian Ka'an. México: Secretaría de Medio Ambiente y Recursos Naturales. 
Julisa Guadalupe Cabrera-Borraz, Lucinda Arroyo-Arcos, Romano Gino Segrado-Pavón, Orlando Colín-Olivares. Instrumentos de gestión de áreas naturales protegidas y participación comunitaria

CONANP. (7 de Junio de 2017). Comisión Nacional de Áreas Naturales Protegidas. Obtenido de conanp.gob: http://sig.conanp.gob.mx/website/pagsig/datos_anp.htm

Cortés Padilla, M. (2012). Metodología de la investigación . México: TRILLAS. Brenner, L., \& Vargas del Río , D. (2010). Gobernabilidad y gobernanza ambiental en México. La experiencia de la Reserva de la Biosfera Sian Ka'an. Polis: Investigación y Ánalisis Sociopolítico y Psicosocial, 115-154.

Diario Oficial de la Federación. (23 de Enero de 2015). Secretaría de Gobernación . Obtenido de Diario Oficial de la Federación: http://www. dof.gob.mx/nota_detalle.php? codigo $=5379437 \&$ fecha $=23 / 01 / 2015$

Fernandes de Santana, V., Fontes Filho, J., \& Barroso Rocha, S. (2015). Gestión local de recursos de uso común en turismo. La perspectiva de Elionor Ostrom. Estudios y perspectivas en turismo, 56-75.

Guillen, A., Sáenz, K., Badii, M., \& Castillo, J. (2009). Origen, espacio y niveles de participación ciudadana. Daena: International Journal of Good Conscience, 179-193.

Hernández Sampieri, R., Fernández Collado, C., \& Baptista Lucio, M. d. (2010). Metodología de la investigación. México: McGraw-Hill.

Instituto Nacional de Ecología . (1996). Programa de Manejo de la Reserva de la Biosfera Sian Ka'an. México.

Instituto Nacional de Estadística y Geografía. (2010). INEGI. Obtenido de INEGI Web site: http://www.microrregiones.gob.mx/catloc/contenido.aspx? refnac $=230080133$

LGEEPA. (4 de Junio de 2012). México, México: Diario Oficial de la Federación.

López Hernández, J. R., Bello Baltazar , E., Estrada Lugo, E. I., Brunel Manse, M. C., \& Ramírez Miranda, C. A. (2012). Instituciones locales y procesos organizativos: el caso de la Reserva de la Biosfera de Sian Ka'an. Estudios Sociales, 67-93.

López Hernández, J. R. (2013). La participación social en la construcción de procesos de gobernanza ambiental en la Reserva Sian Ka'an, Quintana Roo, México. México: El Colegio de la Frontera Sur.

McInnes, R., Kenza Ali, M., \& Pritchard, D. (2017). Ramsar and World Heritage Conventions: Converging towards success. Switzerland: Ramsar Convention Secretariat. 
Mirabal Pérez, Y., \& Flores Pérez, J. I. (2016). Uso público de Áreas Protegidas. Una alternativa para la participación comunitaria. Universidad y Sociedad, 138-145.

Morúa, A. (2010). La participación comunitaria en la gestión ambiental. Revista Venezolana de Economía y Ciencias Sociales, 125-135.

Murillo, J., \& Martínez, C. (30 de Noviembre de 2010). Investigación Etnográfica. Métodos de Investigación Educativa en Ed. Especial. México.

Ortiz Lozano, L. D., Arceo Biseño, P., Granados Barba, A., Salas Moreal, D., \& Jiménez Badillo, M. d. (2010). Zona costera. En E. Florescano, \& J. Ortíz , Atlas del patrimonio natural, histórico y cultural de Veracruz (págs. 123-146). Veracruz: Gobierno del estado de Veracruz y Universidad Veracruzana.

Ostrom, E. (2011). El gobierno de los bienes comunes. La evolución de las instituciones de acción colectiva. México: Editorial Fondo de Cultura Económica

Paz Salinas, M. F. (2008). De áreas naturales protegidas y participación: convergencias y divergencias en la construcción del interés público. Nueva antropología, 51-74.

Pérez Calderon, J. (2010). La política ambiental en México: Gestión e instrumentos económicos. El cotidiano, 91-97.

Schmidt, G. (2010). Territorios indígenas y Áreas Protegidas. Servicio de las Iglesias Evangélicas en Alemania (EDD), 10-26. 